\title{
AdenofibromeGeant Des Seins Axillairesbilateraux Chez Une Femme En Post-Partum
}

\author{
Kabulukadingi Arnauld ${ }^{1 *}$, MwilambweNgoy Steve ${ }^{1}$, AmaniMaleya Chuma $^{1}$, \\ Kabila Mfyama Marcel ${ }^{1}$, PatauleFatuma Honorine ${ }^{1}$, KalengaMwenzeKayamba $^{1}$. \\ ${ }^{I}$ Département De GynecologieEt Obstétrique, Faculté De Médecine/Université De Lubumbashi, RD Congo.
}

\begin{abstract}
We report on a case of a giantfibroadenoma in a 22-years-old women in whomsurgical ablation of a bilateralaxillary mass histologicaldocumented as accesorybreasts. Throughthis observation and review of the literature, wedescribeclinicalfeatureswhichcan orient clinician to a diagnosis of supernumeraryaxillarybreast and emphasizethat, pathologies in normal locatedbreastcanoccur in a supernumerary one.
\end{abstract}

Keywords : Axillarybreast, fibroadenoma, post-partum.

\section{Introduction}

Le sein surnuméraire est un sein sus du nombre normal (deux) se développant sur la ligne lactéale. Les seins surnuméraires siègent dans $90 \%$ au thorax, $5 \%$ à l'abdomen, et $5 \%$ à l'aisselle [1].

Chez les mammifères le développement du sein commence dès la quatrième semaine de vie intra-utérine. Il se fait par la formation de la crête mammaire ou ligne lactéale. Il s'agit d'un épaississement de l'ectoderme de chaque côté de la face ventrale de l'embryon, allant de la future aisselle à la future aine. Le long de cet épaississement, apparaissent des bourgeons mammaires par paires symétriques. Ces bourgeons sont pectoraux chez l'homme et subsistent après la disparition de la crête mammaire à la septième semaine. Cependant, le long de la crête mammaire, peuvent apparaitre d'autres bourgeons dont la persistance aboutit aux seins accessoires ou surnuméraires [1].

Si le diagnostic en est facile en présence d'un mamelon et d'un écoulement lacté, il devient plus difficile en leur absence ou en cas de prédominance du tissu graisseux. Ainsi la confusion avec toutes les étiologies des masses axillaires est-elle possible [2].

L'objectif de cette observation est de montrer les caractéristiques cliniques d'une masse axillaire pouvant orienter le clinicien vers le diagnostic d'unsein axillaire, mais aussi de souligner la survenue, sur ces seins, des pathologies observées sur des seins de localisation normale au cours de la gravido-puerperalité.

\section{Observation}

Mme K. 22 ans, primipare nous est transférée le 26 juin 2007, par CRS MSF-B/Mitwaba, pour un cancer du sein droit avec métastases axillaires bilatérales. Ses antécédents n'ont révélés rien de contributif. La patiente a fait remarquer qu'elle portait depuis son enfance deux petites masses axillaires bilatérales, mais c'est au cours de la grossesse que ces deux petites masses ont commencé à prendre du volume jusqu'à un maximum à environ 29 semaines d'aménorrhée (SA) ; et elle nous est transférée au troisième jour post partum.

A notre examen clinique, la patiente était asthénique, pesant $50 \mathrm{Kg}$, ayant $1,64 \mathrm{~m}$ de taille et déshydratée au plan $\mathrm{B}$. Au thorax nous notons ce qui suit : d'une part, deux seins de localisation normale dont celui de la droite est plus volumineux et inflammé par rapport au sein controlatéral qui lui, est normal et fait couler le lait normalement, d'autre part, deux masses axillaires bilatérales d'environ $21 \mathrm{Cm}$ de diamètre, sensibles, n'adhérant pas au plan profond. Nous avons noté d'une part, au sein axillaire droit, deux ulcérations dont la plus importante prenait les deux tiers du cadran infero-interne de ce sein et une autre située à l'intersection des cadrans supero-externe et infero-externe intéressant le quart de chacun de ces deux cadrans (Fig. 1). Ces ulcérations faisaient sourdre du pus jaunâtre et bien lié ; d'autre part, au sein axillaire gauche une ulcération intéressant les quatre cadrans et prenant environ les trois quart de toute la surface de ce sein (Fig.2) et qui faisait sourdre du pus de même caractéristiques macroscopiques que celui du sein axillaire controlatéral. Ces deux seins axillaires n'ont pas d'aréoles mammaires. L'examen cardio-pulmonaire et abdominal est normal. L'examen obstétrical bimanuel montre un col utérin perméable, un utérus en involution et indolore, les annexes totalement libres et le doigtier ramène des lochies séro-sanglantes.

A l'issue de notre examen clinique nous émettons comme hypothèses diagnostiques: seins surnuméraires axillaires bilatéraux avec mastite puerpérale suppurée ou lipomes axillaires géants bilatéraux suppurés associés à une mastite puerpérale du sein droit.

Une réhydratation a été instaurée associée à une antibiothérapie faite d'azithromycine $10 \mathrm{mg}$ par kilogramme de poids corporel par jour pendant deux jours et une ablation chirurgicale de ces masses axillaires a 
été réalisée deux jours après avoir remonté l'état général et fait un bilan préopératoire complet. L'exérèse chirurgicale laborieuse a duré trois heures et demi et l'intervention était très hémorragique. Aucune adénopathie satellite n'a été notée au niveau des creux axillaires en per-opératoire. La masse tumorale droite à pesé 3400 grammes et celle du sein gauche 3050 grammes (Fig. 3) et les suites post opératoires étant normales, la patiente est sortie de l'hôpital au $15^{\text {ième }}$ jour pour être suivie en ambulatoire. Les masses ont été envoyées au laboratoire de diagnostic cytohistopathologique qui donne les résultats suivant :a) les masses montrent une prolifération mixte glandulaire et fibroblastique. b) de nombreuses formations kystiques de grande taille. En somme, il s'agit d'un fibroadénome géant bilatéral des seins.

A la lumière des renseignements cliniques et paracliniques, nous retenons en définitive le diagnostic d'adénofibrome géant des seins axillaires bilatéraux et la patiente a été soumise sous traitement fait d'une antibiothérapie à la céfotaxime pendant 5 jours

\section{Discussion}

Le sein surnuméraire est un sein sus du nombre normal (deux) se développant sur la ligne lactéale. Les seins surnuméraires siègent dans $90 \%$ au thorax, $5 \%$ respactivement à l'abdomen, et à l'aisselle [1]. Dans ce cas, les masses sont bilatérales et symétriques contrairement au lipome. Les difficultés de diagnostic clinique de ces seins surnuméraires chez notre patiente trouvent des explications anatomiques et physiologiques. L'absence d'aréole explique que ces tumeurs n'orientent pas d'emblée vers un sein surnuméraire. Le lait ne pouvant s'évacuer, provoque un engorgement mammaire responsable de l'augmentation considérable du volume des tumeurs après l'accouchement et pendant la lactation. Le lipome axillaire est une situation rare que de Andrade a noté un cas sur 31 masses axillaires [2]. Il se présente comme une masse sous-cutanée, de consistance élastique, à contours réguliers, indolore ou très peu sensible, mobile par rapport aux plans profond et superficiel.

De formation embryologique cutanée, le sein est solidaire à la peau. Ainsi les seins surnuméraires ne sont pas mobiles par rapport à la peau. En revanche il existe un plan de décollement rétroglandulaire. C'est un plan de glissement entre la couche graisseuse retromamelanaire dont la face profonde est limitée par le fascia transversalis et l'aponévrose de recouvrement du muscle sous-jacent [3]. Ceci rend le sein indépendant du muscle sous-jacent par rapport auquel il est mobile.

La controverse persiste quant à l'attitude thérapeutique : si certains auteurs préconisent l'abstention en dehors de complications $[4,5]$, d'autres optent pour une exérèse systématique, L'exérèse permettrait de prévenir les complications telle la dégénérescence maligne [6]. L'incidence de cette cancérisation paraît basse : Chiari l'évalue à $0,3 \%$ de tous les cancers du sein [7].deCholnoky pour sa part note une cancérisation de sein surnuméraire axillaire 3,8\% [8]. La littérature rapporte en outre des cas de seins surnuméraires pris pour une hydrosadénite suppurée [9] ou une adénopathie satellite d'un cancer de sein [10].Par ailleurs, un cas d'adénome tubaire géant sur sein accessoire situé sur la paroi antérieure du thorax, a été rapporté en chine [11]. Notre attitude a consisté en l'exérèse de toute masse axillaire suivie de l'examen histologique pour en préciser la nature, l'exérèse chirurgicale étant le moyen le plus sûr pour prévenir les complications.

\section{Conclusion}

En somme, les principaux caractères cliniques qui orientent vers les seins surnuméraires plutôt que vers les lipomes axillaires sont : le caractère bilatéral et symétrique de la localisation axillaire, une mobilité par rapport au plan profond et non au plan superficiel, et enfin une augmentation transitoire du volume lors de la grossesse et de la lactation. Cette anomalie congénitale étant rare, il convient de rappeler au clinicien d'y penser lorsqu'il est en présence des masses axillaires.

Conflits d'intérêt: Aucun.

Contribution des auteurs: Tous les auteurs ont participé à la réalisation de ce travail, ils ont lu et approuvé la version finale du manuscrit.

\section{Références}

[1]. Velanovich V. Ectopic breast tissue, supernumerary breasts, and supernumerary nipples. South Med. J., 1995, 88, 903-906.

[2]. de Andrade J.M., Marana H.R., SarmentoFilho J.M., Murta E.F., Velludo M.A., Bighetti S. Differentialdiagnosis of axillarymasses. Tumori, 1996, 82, 596-599.

[3]. Espie M. et Gorins A. Développement et anatomie du sein normal. In le sein, Editions Eska, Paris, 1999. 28-31

[4]. Baruchin A.M., Rosenberg L. Axillary breast tissue: clinical presentation and surgical treatement. Ann. Plast. Surg., 1996, 36, 661662.

[5]. Lesavoy M.A., Gomez-Garcia A., Nedl R., Yospur G., Syiau T.J., Chang. Axillary breast tissue: clinical presentation and surgical treatement. Ann. Plast. Surg., 1995, 35, 356-360.

[6]. Markopoulos C., Kouskos E., Kontzoglou K., Gogas G., Kyriakou V., Gogas J. Breast cancer in ectopic breast tissue. Eur. J. Gyneco. Oncol., 2001, 22, 157-159.

[7]. Chiari H.H. Zurfrage des karzinonomas in aberrantembrunstdrusengewebe. Bruns. Beitr. Klin. Chir., 1958, 197, $307-314$.

[8]. deCholnoky T. Accessory breast tissue in the axilla. NY State J. Med., 1951, 51, 2245-2248

[9]. Silverberg M.A., Rahman M.Z. Axillary breast tissue mistaken for suppurativehydrosadenitis: an avoidable error. J. Emerg. Med., 2003, 25, 51-55. 
[10]. Maiorano E., Mazzarol G.M., Pruneri G., Mastropasqua M.G., Zurrida S., Orvieto E., Viale G. Ectopic breast tissue as possible cause of false-positive axillary sentinel lymphnode biopsies. Am. J. Surg. Pathol., 2003, 27, 513-518

[11]. Yaoyu Huang, Hao Zhang, Qian Zhou, Lijun Ling and Shui Wang, Giant tubular adenoma of the accessory breast in the anterior chest wall occurred in a pregnant woman. Diagnostic Pathology, 2015,10:60

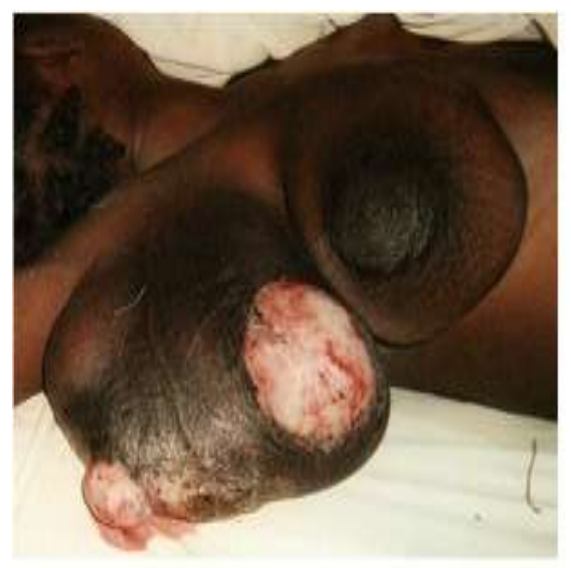

Fig. 1: Sein axillaire droit ulcéré

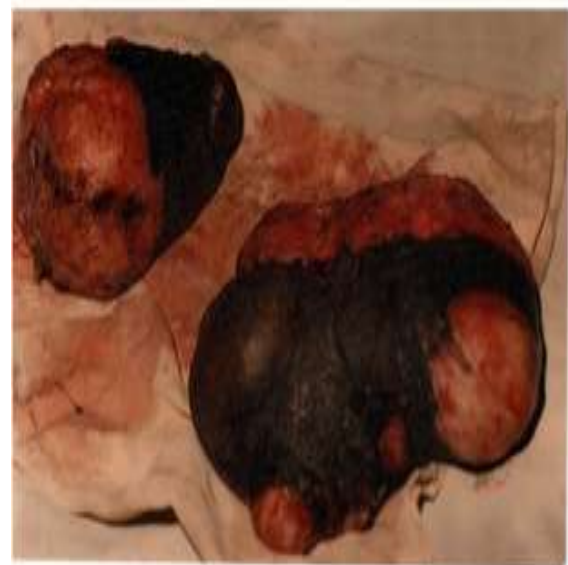

Fig. 3: Deux seins axillaires

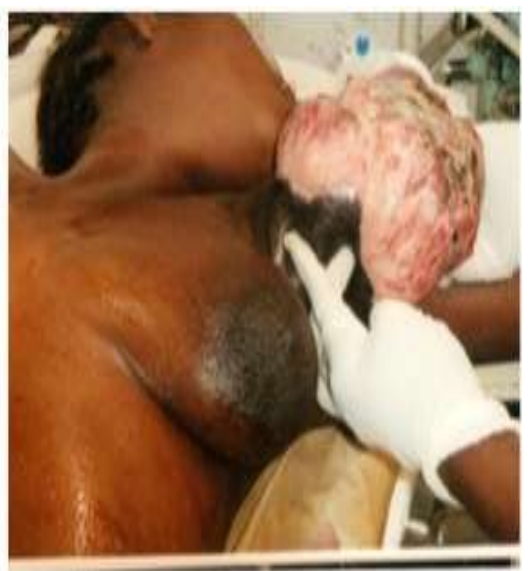

Fig. 2: Sein axillaire gauche ulcéré

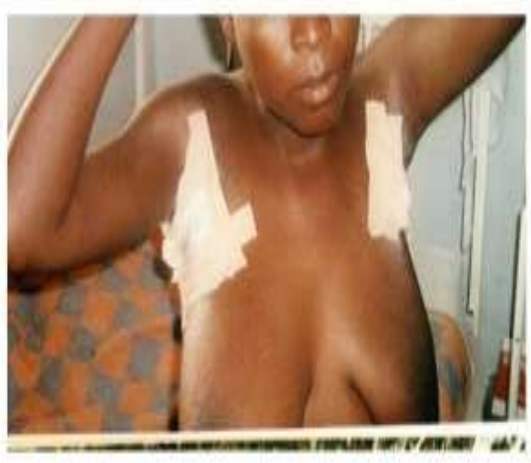

Fig. 4: patiente au sixième jour post opératoire 